\title{
CLINICAL FINDINGS AND MANAGEMENT OF CHRONIC BLEPHARITIS IN A 25-YEAR OLD FEMALE -A CASE REPORT
}

BY

\author{
*OSAIYUWU, A. B. AND EBEIGBE, J. A. \\ DEPARTMENT OF OPTOMETRY, FACULTY OF LIFE SCIENCES, \\ UNIVERSITY OF BENIN, BENIN CITY, NIGERIA. \\ EMAIL: bosaiyuwu@yahoo.com \\ *Corresponding author
}

\section{ABSTRACT}

Blepharitis is an inflammation of the eyelid margins which sometimes leads to secondary changes in the conjunctiva and cornea. Symptoms of blepharitis tend to be longstanding, thus, blepharitis is more of a chronic condition than being acute and both eyes are usually affected, however, the symptoms can easily be eased. Once symptoms have eased, daily 'eyelid hygiene' can usually keep the symptoms to a minimum. Vision is rarely affected. This case report reviewed the management of blepharitis in a 25-year old girl the clinical findings and treatment options were also discussed.

KEYWORDS: Blepharitis, Madarosis, Conjunctivitis, Keratitis, Staphylococcus auerus.

\section{Received 03/03/2010}

\section{INTRODUCTION}

Historically, blepharitis has been divided into anterior forms (affecting the anterior lid margin and eyelashes) and posterior forms (affecting the meibomian glands) ${ }^{1,2}$. Blepharitis is one of the most common eyelid disorders often associated with tear film disruption and accompanying symptoms of which the mainstay of management is meticulous lid hygiene not medicinal ${ }^{3}$. It is more common in young females ${ }^{4}$. One of the most accompanying symptoms is madarosis of which infection due to Staphylococcus aureus is one of the causes. Long-standing staphylococcal infection is associated with madarosis, whitening (poliosis) and misdirection (trichiasis) of eyelashes ${ }^{5}$. Blepharitis is characterized by inflammation of the eyelid margins. It may cause redness of the eyes, itching and irritation of the eyelids in one or both eyes. Its appearance is often confused with conjunctivitis and due to its recurring nature; it is the most common cause of 'recurrent conjunctivitis' in older people. It is also often mistaken for 'dry eye' by patients due to the gritty sensation that may occur.

\section{CASE REPORT}

A 25-year old female patient, IU presented to our Optometry Clinic on 30 April 2008 with a history of irritation in both eyes since the past eighteen months. She reported that the problem started mildly with occasional discharge and itching, but progressed to a more copious discharge, itching, redness and almost a complete loss of eyelashes (madarosis).She also reported that she applied a couple of over-the-counter

\section{Accepted 04/06/2010}

(OTC) drugs of which she could only remember chloramphenicol eye drop as one of the drugs applied. The patient had never had an eye examination prior to her visit to our clinic. The patient also complained that the symptoms often 'come and go' and that occasionally the symptoms were so very severe and there were also long periods without any symptoms. The patient's medical and family history was negative.

\section{CLINICALFINDINGS}

Her unaided visual acuity at distance was OD $6 / 6$, OS $6 / 6$ and OU $6 / 5^{+}$, while her unaided visual acuity at near $(0.4 \mathrm{~m})$ for OD and OS was N5.

Colour vision testing with pseudoisochromatic plates was normal OU.

Pupils were equally round and reactive to light, no afferent pupil defect was noted OU. Confrontation fields were full to finger count OU. Extraocular muscles were unrestricted in all gazes and the alternate cover test demonstrated both orthophoria at distance and near.

Both eyes looked generally unhealthy in appearance; the eyelids were inflamed and sore with the eyes having some discharge, irregularity and ulceration of the lid margins and madarosis and there was improper lid closure when the patient was instructed to close the eyes.

Slit lamp examination revealed the following: Lashes: loss of lashes (madarosis), scanty and misdirection of lashes (trichiasis) and crusting of lashes.

The meibomian orifices: crusting and plugging. 
The conjunctiva: papillary injection.

Corneal findings: punctate epithelial erosions, marginal infiltrates, pannus and phlyctenule formation.

Direct Ophthalmoscopy: no abnormality; both crystalline lenses were transparent, there were normal optic nerves with a cup-to-disc ratio of 0.3/0.3 OU, neuroretinal rims were healthy, retinal vessels were normal with an arterio-venous ratio of $2 / 3$ OU and both eyes presented with normal macular findings.

\section{Diagnosis: Chronic blepharitis}

IU was counseled and instructed to go for a swab test and was scheduled to be seen on 5 May 2008.

\section{FOLLOW UP\#1}

IU returned on 5 May 2008 to review the swab result. The swab result revealed the causative organism as Staphylococcus aureus. Her visual acuity remained stable with unaided acuities of $6 / 6$ $\mathrm{OD}$ and $\mathrm{OS}$ and $6 / 5^{+} \mathrm{OU}$. Schirmer test was carried out on the patient and the result was quite favourable.

IU was counseled on the treatment regimen that would be given and that a strict compliance was all that would be needed for an effective treatment. She was also made to realize that the treatment may span through months and thus she has to learn to be patient. She was instructed to carry out the treatment thus:

$\square$ Firstly, the application of heat to warm the eyelid gland secretions and to promote evacuation and cleansing of the secretory passages.

$\square$ Secondly, the eyelid margin should be washed mechanically to remove adherent materials such as collarettes and crusts and to clean the gland orifices, water often is used. Gentle mechanical scrubbing of the eyelid margin is all that is needed, vigorous scrubbing is not necessary and may be harmful.

$\square$ Thirdly, the medications should be applied to the eyelid margin after it has been soaked and scrubbed.

The patient was placed on

- Cap. Doxycycline 200mg stat, then 100mg for 2 weeks.

- Ointment Erythromycin (0.5\%), 0.5inch ribbon to be applied to lid margin qid for 2 weeks.

- Sulfacetamide sodium and prednisolone acetate (Blephamide; $10 \%$ sulfacetamide; $0.2 \%$ prednisolone), 1 drop qid for 2 weeks.
- $\quad$ The few misdirected lashes were epilated after instillation of two drops of $0.05 \%$ novesine.

- Tab. Vit. C, 2 tabs tds for 2 weeks was also prescribed.

The patient was again reminded to be drug compliant and to return in 2 weeks time for follow up.

\section{FOLLOW UP\# 2}

The patient returned for follow up on 19 May 2008. Her visual acuities remained the same and she reported that she had been drug compliant. The general appearance of the eyes looked improved when compared to her first visit.

Slit lamp examination was conducted on her and findings were as listed;

Absence of misdirected lashes

There was reduced crusting of the lashes

There was a marked reduction of crusting and plugging on the meibomian orifices

There was almost a total absence of conjunctival papillary injection.

The cornea still presented with some punctate epithelial erosions, a few marginal infiltrates and the pannus was resolving quietly as well as the phlyctenules.

Direct ophthalmoscopy revealed no abnormality.

The patient was encouraged to keep up with the compliance as the prognosis was good. Her medications were reviewed and she was advised thus:

Take: Cap. Doxycycline 100mg for another 2 weeks

Gutt Blephamide 1 drop bid for 2 weeks

Oint. Erythromycin $(0.5 \%)$ qid for 2 weeks

Tab. Vit.C 2 tabs tds for 2 weeks

Patient was counseled to continue cleaning the lids as instructed and generally maintain a very good hygiene. IU was told to report again to the clinic in another 2 weeks time for follow up.

\section{FOLLOW UP\# 3}

IU returned for follow up on 2 June 2008. Her vision was unchanged and the eyes looked quite healthy. The patient said that she was happy with the drastic improvement of her eyes. Slit lamp examination revealed absence of misdirected lashes, a few crusts on the lashes, a few crusts and absence of plugging on the meibomian orifices, absence of conjunctival papillary injection, pannus and phlyctenules had completely resolved but the 
cornea still presented with a few punctate epithelial erosions and marginal infiltrates.

The patient was commended for not only being drug compliant but also in maintaining good lid hygiene. Her medications were reviewed thus:

Take: Cap.Doxycycline 100mg for another 2 weeks

Gutt Blephamide 1 drop daily for 2 weeks

Oint. Erythromycin $(0.5 \%)$ tds for 2 weeks

Tab. Vit. C 2 tabs tds for 2 weeks.

The patient was told to come for follow up in another 2 weeks time when we hoped the eyes would be fit enough to undergo the test for refraction.

\section{FOLLOW UP\# 4}

IU returned to the clinic on 16 June 2008 . Her vision was unchanged and her eyes were looking really healthy. Slit lamp examination revealed no abnormality and there was also no abnormality detected on ophthalmoscopy.

IU was so very grateful but she wanted to know if she would grow the lashes again. She was reassured that the lashes would re-grow but that it was not going to be spontaneous. We also counseled IU about referring her to an ophthalmologist to carry out lid repairs, but she was not so enthusiastic about it and she rejected the idea of the referral. We also made her to realize that the treatment had not ended meaning that she should still continue with the lid hygiene religiously. We reviewed her medication by placing her on the doxycycline, 100mg daily for the next 2 weeks; she was to stop instilling the blephamide but to continue with the ointment erythromycin to be applied twice a day for another 2 weeks. Her next follow up was in a month's time, 16 July 2008.

The retinoscopic findings were:

OD $+0.75 /-0.50 \times 180$

OS $\quad+0.75 /-0.25 \times 180$

The subjective refraction findings were same as retinoscopic findings. The patient was given another appointment to check her progress and possibly prescribe glasses, but she never returned. patients with chronic blepharitis include Staphylococcal epidermidis, Propionibacterium acnes, corynebacteria and Staphylococcus aureus. ${ }^{6,7}$. A staphylococcal infection normally presents predominantly in young women to middle-aged women ${ }^{4}$. Collarettes or cuffs of fibrin (matted, hard scales) extend from the base of and along lashes as a sleeve. Eyelid ulcerations are fine at the base of lashes. There is also the absence of eyelashes in staphylococcal infection; this condition is very rare in seborrheic infection.

The management of blepharitis is a process which must be carried out for prolonged periods of time, implying that even when symptoms have subsided, the treatment should still continue but at reduced doses. Prognosis is usually good when the patient is compliant to the instructions given as per maintenance of proper lid hygiene ${ }^{3}$. The patient in this report was very compliant and it reflected in the prognosis report.

Conjunctivitis and keratitis can result as a complication of blepharitis and requires additional treatment besides eyelid margin therapy. Antibiotic-corticosteroid solution can greatly reduce inflammation and symptoms of conjunctivitis. Corneal infiltrates can be treated with antibiotic-corticosteroid drops ${ }^{1}$. Small marginal corneal ulcers can be treated empirically, but larger paracentral ulcers should be scraped and specimens sent for diagnostic slides and for culture and sensitivity testing ${ }^{4}$.

Overall the prognosis for patients with blepharitis is good to excellent. Patients with unilateral or very asymmetric blepharitis may have sebaceous cell carcinoma. An oculoplastics consult may be required for a lid biopsy ${ }^{4,7}$.

This case demonstrates the role of patient history, clinical observation and swab study in the diagnosis of blepharitis. In most cases, the diagnosis can confidently be made without a swab test, however, the information derived from the swab test is critical as knowledge of the causal organism would aid the optometrist into a pool of medications that such organism(s) is (are) susceptible to. Although prognosis for patients with blepharitis is generally favourable, patients should continue to self-monitor their vision even after resolution.

\section{DISCUSSION}

The most common organisms isolated from 


\section{REFEREN CES}

1. Jackson, B. W. (2008): Blepharitis: current strategies for diagnosis and management. Can. J. Ophthalmol, 43:170-9.

2. Riordan-Eva, P. and Whitcher, J. P. (2004): Vaughan and Asbury's General Ophthalmology.16 ${ }^{\text {th }}$ Edn. McGraw Hill Company, Lange, pp. 19-26.

3. Ron, M. and Randall, T. (2004): Blepharitis. Clin. Refr. Optom, 15 (4):110-2.

4. Sunita, A., Athiya, A., David, J. A., Lucio, B., Jorge, L. A., Suresh, K. P. and Amar, A. (2002):
Textbook of Ophthalmology. $1^{\text {st }}$ Edn. Jaypee Medical Publishers, pp. 67-9.

5. Sachdeva, S. and Prasher, P. (2008): Madarosis: A dermatological marker. India J. Dermatol. Venereol. Leprol, 74:74-6.

6. Dougherty, J. M. and McCulley, J. P. (1984): Comparative bacteriology of chronic blepharitis Bri. J. Ophthalmol, 68:524-8.

7. McCulley, J. P. and Dougherty, J. M. (1986): Bacterial aspects of chronic blepharitis. Trans. Ophthalmol. Soc. UK, 105 (3):314-8. 